\title{
Analysis of Oscillation of Propulsion System on Hovercraft - Theoretical Framework and Review
}

\author{
Pham Hong Thanh \\ Faculty of Electrical and Engine Engineering, Waterway Transport College No. 2, Ho Chi Minh City, Vietnam
}

Email address:

hongthanh.dt2@gmail.com

\section{To cite this article:}

Pham Hong Thanh. Analysis of Oscillation of Propulsion System on Hovercraft - Theoretical Framework and Review. International Journal of Transportation Engineering and Technology. Special Issue: Transportation Engineering Technology and Education.

Vol. 6, No. 4, 2020, pp. 128-132. doi: 10.11648/j.ijtet.20200604.14

Received: August 2, 2020; Accepted: September 9, 2020; Published: December 22, 2020

\begin{abstract}
With flexible usability, able to operate both on water and land, hovercraft has a long history of development and is widely used in many countries around the world. In this research, we focus on solving the problem of isolation oscillates when a machine - placed on an isolator - operates the pinwheel to create thrust momentum for hovercraft movements. The main content of the author's master degree research is to study the oscillator system integrated in the engine structure to significantly reduce the vibration from the main engine and the propeller of the air cushion ship. The study focused on building a calculation model to find the main vibration characteristics of the propulsion system - motor - motor support structure integrated with damping system. Main objective is finding out the parameter values: transmission characteristics, specific frequencies of the air-cushion engine structure and damping system of supporting structure which are able to withstand the best vibration isolation. Based on reviewed literature, simulations and real-world tests (using CALFEM and following simulation models) are being performed to determine the optimal technology parameters. The findings and results continue to contribute to the improvement of engine technology in particular and seaplane engineering technology in general as required by standards and regulations.
\end{abstract}

Keywords: Propulsion System, Hovercraft, Air Cushion Vehicle, Waterway Transportation

\section{Introduction}

\subsection{Brieft History of Hovercraft}

Hovercraft has a long history of development. Starting from research of Sir Christopher Cockerell in 1956, the first air-cushion ship is built since 1959, the hovercraft SR. N1. Later on, there are many hovercraft models of Saunders Roe Company those marked the development in size, weight, capacity and speed of hovercraft vehicle. The biggest hovercraft ever built is SR. N4 MK. III Princess Anne (tonnage of 320 tons and length of $56.38 \mathrm{~m}$ ).

This type of air-cushion ship, hovercraft, is researched and widely used by many countries such as: Japan (MV. PP15), Russia (Zubr), China (722-1). The hovercraft could be used for both military and non-military usage on land or river and sea where there has no wharf has been built [1-3].

In Vietnam, American-made patrol hovercraft PACV Model 7232SK5 appeared for the first time in the Southern battlefields in the mid-1960s. The ship is $11.8 \mathrm{~m}$ in length, and 7.1 tons in weight, its total capacity of $1000 \mathrm{HP}$, speed of 60 knots $[4,5]$.

\subsection{Summarization and General Reviews}

With flexible usability, able to operate both on water and land, hovercraft has a long history of development and is widely used in many countries around the world. Unfortunately, in Vietnam, there is no official scientific publication about hovercraft's design concept and manufacture process or manufacture technology and test operation; except, those researches conducted by hovercraft research group of the Ho Chi Minh city University of Technology in Vietnam National University Ho Chi Minh City. The hovercraft researches are received many interests from many organizations and companies due to its flexible usability, can be used for national defense, search and rescue mission and tourism purposes [6-8].

Compared with other ship's relatives, hovercraft has specific computational model, technical design, construction design, manufacturing technologies and different coordinating materials which lead to several differences that 
need to be studied. Among dozens of topics, in this research, we focus on solving the problem of isolation oscillates when a machine - placed on an isolator - operates the pinwheel to create thrust momentum for hovercraft movements.

\subsection{Problem Overview}

In industrial environment, there are many sources of oscillation and shock, including motor driven mills, reciprocating compressors and electric motors. The presence of oscillation problems in those motors often leads to undesirable outcomes as the machine malfunctions, followed by regular and expensive machine maintenance.

Sometimes, the oscillation problems can be solved at the source by theoretical analysis but the price for vibration source-solving technology is very expensive compared to its practical applicability. In case of oscillation problems of recent hovercraft and air cushion motor system, the shaft system is raised so that the shaft and pinwheel propulsion are concentric, which need to be studied.

\subsection{Research Methodology}

1. Literature review: review scientific documents such as books, newspapers, documents and articles related to the topic, in order to create the research database.

2. Software application to simulate and CALFEM [9] which aim to identify optimal technology parameters those ensure vibration isolation according to the simulation test scheme (Figure 1).

\subsection{Scientific Significance and Urgency of Research}

\subsubsection{Scientific Significance}

On scientific aspect, the research handles 2 main scientific problems:

1. Influence of transmission characteristics, specific frequencies, dynamic response of: machine assemblies gearbox structure - pinwheel propulsion on the oscillation solation problem of propulsion system of hovercraft.

2. Influence of the isolated material for the propulsion system of hovercraft. The research provides theoretical and empirical basis for the studies on post-manufactured of oscillating isolation systems for air-cushion vessel in general and contributes more knowledge to scientific database related to hovercraft and engine oscillating problem in Vietnam.

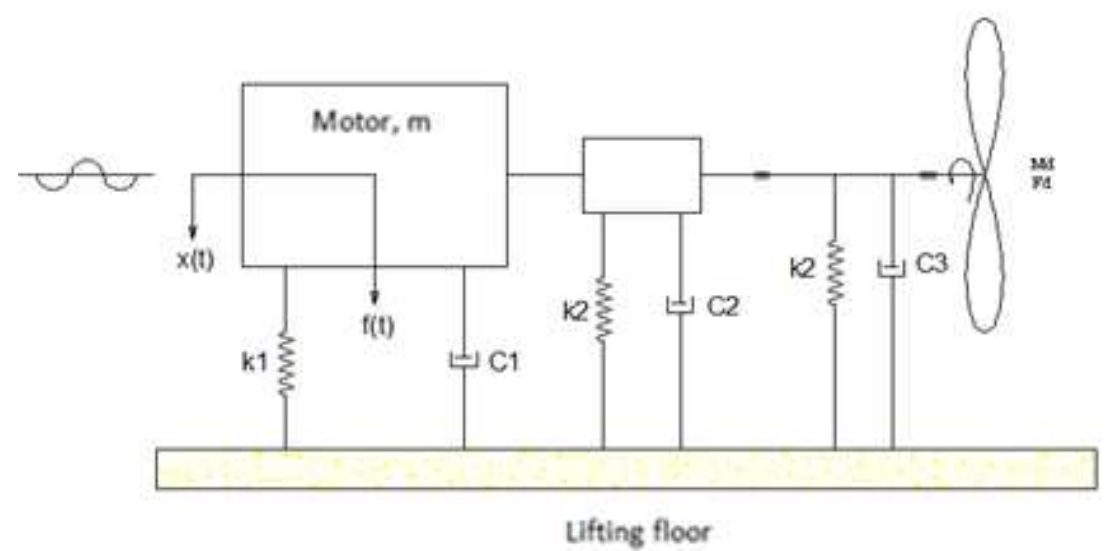

Figure 1. Diagram of tested simulation.

\subsubsection{Urgency of Research}

Because vibration and shaking effect causes significant damage to engine durability, reduces performance and significantly increases maintenance - repair costs when hovercraft is used. In order to increase the efficiency of the air-cushioned vehicle and encourage organizations and individuals to invest in this type of vehicle, studies on oscillation isolation as well as limiting damage caused by engine vibration are essential.

Even through, the problems related to engine vibration can be solved at the source. Unfortunately eliminating vibrations of the motor at the source is very expensive and has a limited applicability which is not appropriate for industrial hovercraft manufacturing, building oscillator isolation systems for engine thrusters becomes a more appropriate solution.

Aiming to solve the engine vibration problem, this research is one of the key factors that could help improving hovercraft manufacturing technology and changing ship construction structure.

\section{Theoretical Framework and Literature Review}

In this section, the research focuses on summarized the literature on oscillation isolation systems of the motor, including models and principles of isolator design [10].

\subsection{Vibration Isolation System}

There are some typical samples of vibration isolation system, described in figure 2 and figure 3.

Figure 2 (a) describes: the source of vibration is the operating motor, the transmission direction from engine to lifting structure. The transmission characteristic $\mathrm{T}$ of the isolator is defined as the ratio of the amount of force infused, 
$f_{t}(t)=k x+c \dot{x}=F_{T} e^{i(\omega t+\alpha)}$, to the stimulus, $f(t)=F_{0} e^{i \omega t}$. The formula is:

$$
T=\frac{F_{T}}{F_{0}}=\frac{\sqrt{1+\left(2 \zeta \frac{\omega}{\omega_{n}}\right)^{2}}}{\sqrt{\left[1-\left(\frac{\omega}{\omega_{n}}\right)^{2}\right]^{2}+\left(2 \zeta \frac{\omega}{\omega_{n}}\right)^{2}}} .
$$

Figure 2 (b) describes: The direction of motion transmission is from the lifting structure through the isolator to the device. In this case, the reason for using the isolator is to protect the measuring instruments against the vibration of the oscillating floor. The transmission characteristic of the isolator is theoretically calculated as:

$$
T=\frac{X}{Y}=\frac{\sqrt{1+\frac{1}{Q^{2}}\left(\frac{f}{f_{0}}\right)^{2}}}{\sqrt{\left[1-\left(\frac{f}{f_{0}}\right)^{2}\right]^{2}+\frac{1}{Q^{2}}\left(\frac{f}{f_{0}}\right)^{2}}},
$$

The result is similar to the case 1 (a).

In additional, when the machine is placed on the isolator, the static weight $\mathrm{W}$ of the device causes the isolator to sunken down a static deflection $\boldsymbol{\delta}_{\text {st. }}$.

Since the specific frequency does not have the resistance of the system of 1-degree of freedom, therefore, the expression for calculating the frequency is:

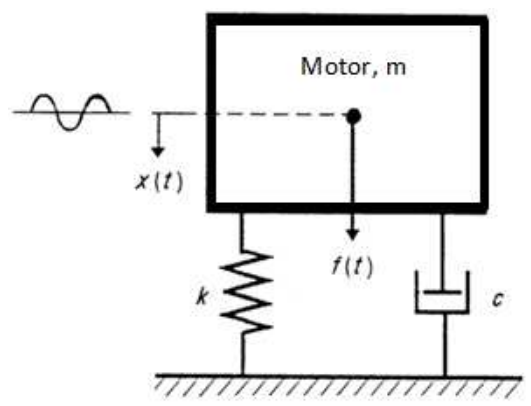

Thin floor (a)

$$
f_{0}=\frac{1}{2 \pi} \sqrt{\frac{k g}{W}}
$$

and

$$
f_{0}=4,98 \sqrt{\frac{1}{\delta_{s t}}}
$$

In conclusion, the specific frequency of a system can be determined by measuring the level of static deflection $\delta_{s t}$. This is only applicable if the spring is linear type and the material of the isolator has the same elastic properties with both static and dynamic conditions.

In fact, most manufacturers of isolators do not often give technical data in terms of hardness; instead, they often provide static deflection data $\delta_{s t}$ corresponding to a certain static load.

$$
T=\frac{F_{T}}{F_{0}}=\frac{m_{2}}{\left(m_{1}+m_{2}\right)}\left[\frac{1}{1-\left(\omega / \omega_{2}\right)^{2}}\right],
$$

with $\omega_{2}=\left[\left(m_{1}+m_{2}\right) k / m_{1} m_{2}\right]^{1 / 2}$ is the specific frequency of the system.

$$
T=\frac{F_{T}}{F_{0}}=\frac{k Z(\omega)}{Z(\omega)\left(k-m_{1} \omega^{2}\right)-k m_{1} \omega^{2}},
$$

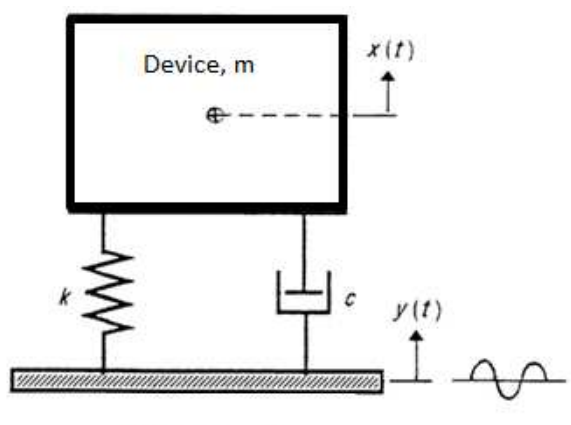

Lifting floor (b)

Figure 2. Models of oscillating isolation system.

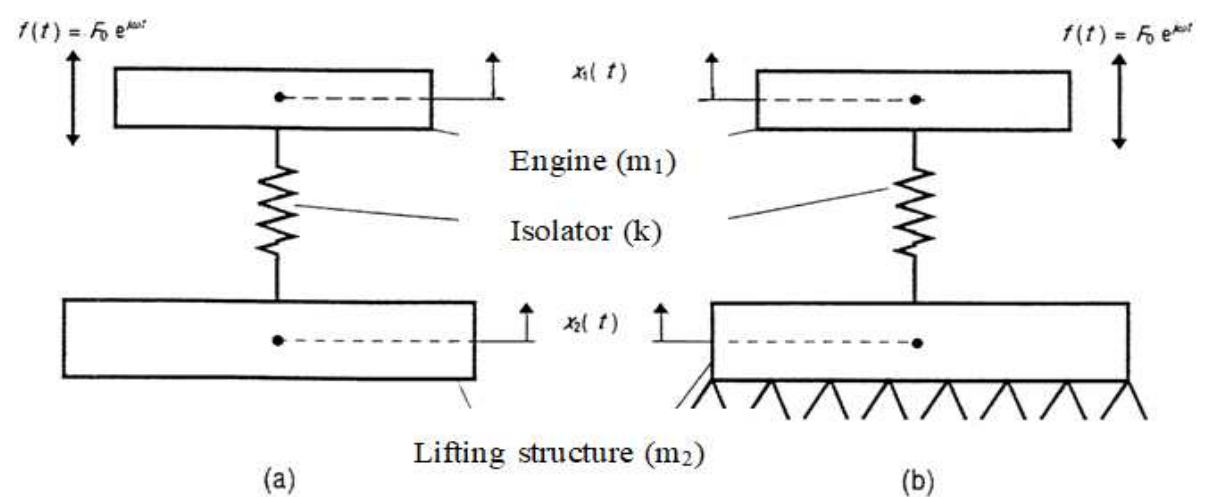

Figure 3. Models of machine with isolator on (a) flexible platform and (b) local flexibility platform. 


\subsection{Elastomers System}

These isolators are made primarily of rubber and synthetic rubber such as neoprene. Generally, rubber isolators are used to isolate small electrical equipment, machines and electronics from relatively high coercive frequencies (see figure 4).

In a controlled environment, rubber is probably the best and most economical insulation material. The rubber itself has dampers properties so it is very practical if the machine operates near the resonant or undergoes the resonance zone during start-up or shutdown. Within a harsh environment, synthetic rubber would be a better option [11].
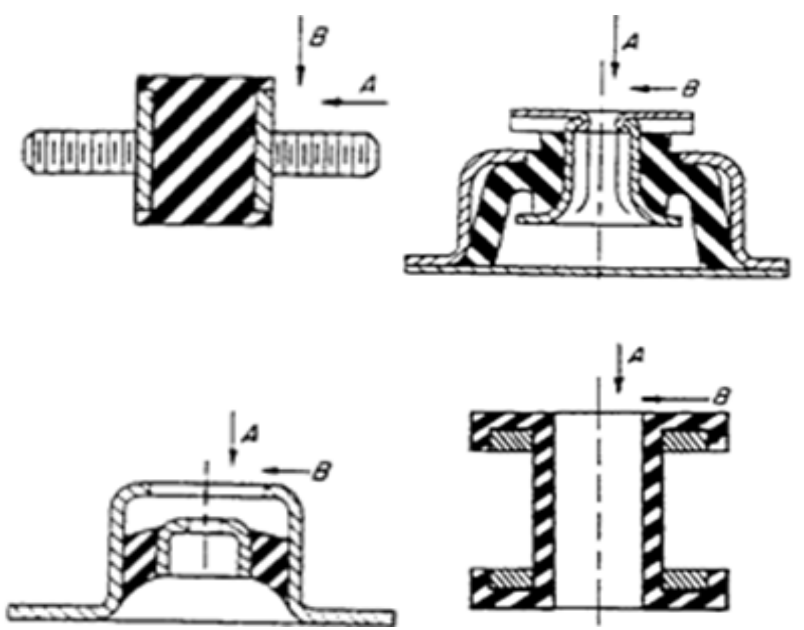

Figure 4. Some typical rubber isolators.

\subsection{Metal Springs}

In industry, metal springs are widely used for oscillating isolation. The metal springs can be used in multiple cases: light, flimsy equipment to heavy industrial machinery (see figure 5).

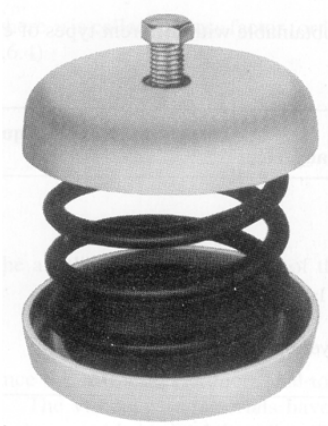

Figure 5. Metal spring isolator model [12].

\subsection{Buffer Isolation System}

Materials for this isolator category include: cork, felt and fiber glass. In general, these are easy to use and organize. They are sold in sheets, which can be cut out according to use, and can be stacked in piles to get different levels of isolation. The Typical damping factor of felt and cork is 0.06 .
Cork has good resistance to corrosion and solvents, and relatively insensitive to a wide range of temperatures. Some felt pads are made of organic material which should not be used in an industrial environment (where uses solvents). Fiberglass pads, on the other hand, resist well to industrial solvents.

\subsection{Pneumatic Systems}

This type of isolation uses the principle of supporting a static load on an air column. This isolator is especially useful for systems that require very low resonant frequencies, within the range of 0.5 to $3 \mathrm{~Hz}$.

An air spring causes the system to achieve 0 static deflections under the load. This is particularly noticeable because a common spring system needs to deflect $1 \mathrm{~m}$ to obtain a specific frequency of $0.5 \mathrm{~Hz}$, and nearly $3 \mathrm{~cm}$ for a specific frequency of $3 \mathrm{~Hz}$.

The equation for determining $\mathrm{f}_{0}$ of a raised mass on an air column is:

$$
f_{0}=\frac{1}{2 \pi} \sqrt{\frac{\gamma A g}{V}}
$$

\subsection{Principles of Isolator Design}

The isolation system should be designed so that the specific frequency of the lifting structure is always three to five times the specific frequency of the isolation system. In such cases, the lifting structure is considered as a rigid element of the system and is not oscillated.

In oscillating isolation, the amplitude of oscillation is usually quite small and the nonlinearity of the isolator is usually not important unless the level of deflection created by the fixed weight is relatively large. In the practical application of the isolator, some considerations should also be considered.

First, it is important that the isolators are properly positioned for the machine's center of gravity.

Second, the center of the machine should be as low as possible. If the swinging effect, or other instability, becomes a serious problem, the effective center of gravity can be further lowered by placing the machine on a heavy weight and then isolate mass-and-machine system.

Third, it is possible to use a multi-clutch isolation system, that is the separation between the machine and the mass and between the mass and its foundation structure, in order to obtain the transmission characteristics that allow more reduction of frequency components at on polynomial system resonances, but the design of composite systems is complex.

\section{Conclusions}

This scientific article is the first part of the whole research project [13] about the oscillation of propulsion system on hovercraft, which focuses on project overview and theoretical framework and review. 
First, this article summarized the brief scientific information, such as: research introduction, history of hovercraft and applied methodology. Next, related scientific documents, articles, studies are reviewed and summarized, which focus on reviewing on different types of isolators which are applied to oscillation isolation problems and principles of isolator design.

Based on the reviewed literature, the simulation and practical test (used CALFEM and followed the simulation model), which aim to identify optimal technology parameters, could be started. The findings and results further contribute to the improvement of engine technology in short and hovercraft technology and manufacture in general as required in Standards and Rules [14-16].

\section{Acknowledgements}

Author would like to acknowledge Assoc. Prof. Le Dinh Tuan at the Ho Chi Minh city University of Technology (HCMUT) as thesis advisors of this research, and author is gratefully indebted to him for his very valuable comments on this researh. Author is also grateful to the support from the Shipbuilding Engineering Lab. of the HCMUT to undertake his Master thesis.

\section{References}

[1] Yun, Liang, and Alan Bliault. Theory and design of air cushion craft, Elsevier, 2000.

[2] Saunders, Stephen (RN). Jane's Fighting Ships 2003-2004, Jane's Information Group, 2003. ISBN 978-0-7106-2546-5.

[3] Zubr Class (Pomornik), Naval Technology. [on-line] navaltechnology.com.
[4] Keaveney, Kevin. Cavalry Afloat: The 39th Cavalry Platoon in the Mekong Delta. Armor. Fort Knox: US Army Armor Center. (July-August 1993).

[5] Singh, Risaldar. Air Cushion Cavalry: The US Army's Bell SK5 ACV in Vietnam. One Project Too Far, (May 2015).

[6] Le Dinh Tuan. Research on manufacture of small ACV”, (Proj. B2010-20-10TÐ), VNU-HCM, 2010.

[7] Le Dinh Tuan, Tran Nguyen Nguyen Khoi, Nguyen Tri Dung, Nguyen Huu Toan. Generality on air cushion vehicle design and applications. Science \& Technology Development, Vol 12, No. 14-2009, 2009, pp. 12-19.

[8] Le Dinh Tuan, Doan Hien. On the design of search and rescue hovercrafts. Science \& Technology Development, Vol 18, No. K7- 2015, 2005, pp. 126-135.

[9] CALFEM, a finite element toolbox -Version 3.4, Lund University, 2004.

[10] William T. Thomson. Theory of Vibration with Applications (4th Ed.). Department of Mechanical and Environmental Engineering, University of California, Santa Barbara, California, 1993.

[11] dB Engineering. Isolator Selection Guide. [on-line] https://novibration.com/.

[12] Free standing spring $\quad-\quad$ isolator. [on-line] https://flexonics.com.

[13] Pham Hong Thanh. Analysis of oscillation of propulsion system on hovercraft. MSc thesis, HCMUT/VNU-HCM, 2020.

[14] American Bureau of Shipping. Guidance Notes On Ship Vibration, 16855 Northchase Drive Houston, TX 77060 USA, 2006.

[15] DNV GL, Pt. 4 Sec. 1.8.2, Measurements of vibration, 2019.

[16] ISO 1940/1, Permissible residual specific unbalance for different quality grades $G, 1973$.

\section{Biography}

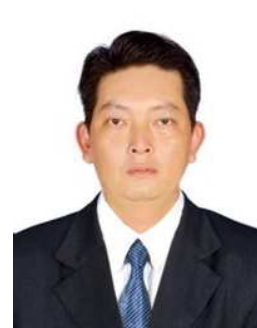

Pham Hong Thanh (born in 1975, Ho Chi Minh City). Since receiving his degree as an Engineer of Shipbuilding Engineering (1998, Nha Trang University), he has been a lecturer in the Faculty of Electrical and Machinery, College of Waterway Transport 2, Ho Chi Minh City, Vietnam. In 2020, Mr. Thanh graduated with a Master of Engineering in Mechanical Transportation Engineering, majoring in Marine Engineering at the University of Technology, VNUHCM, Vietnam. 